\title{
EMOCIÓN Y MOTIVACIÓN EN TEA Y ATENCIÓN TEMPRANA
}

\author{
Rocío García Pascual \\ Universidad de León \\ rocio.garcia@unileon.es \\ Alfredo Rebaque Gómez \\ Universidad de León \\ Ma Ángeles García Mata \\ Universidad de León \\ Jana Blanco Fernández \\ Universidad de León \\ Ana $\mathbf{M}^{\mathrm{a}}$ de Caso Fuertes \\ Universidad de León
}

Fecha de Recepción: 18 Enero 2019

Fecha de Admisión: 30 Abril 2019

\section{RESUMEN}

La motivación y la emoción son elementos fundamentales para el aprendizaje. Cuando hablamos de desarrollo temprano y TEA, estas dos dimensiones adquieren un mayor peso debido a los déficits socioemocionales, los intereses restringidos y las conductas estereotipadas que hacen que no se vinculen de igual forma a adultos y a iguales. Existe amplia evidencia de los beneficios de iniciar una intervención especializada en TEA, aun cuando sólo hay signos de alerta que pueda repercutir en la mejora de los síntomas del trastorno (Dawson, 2008). El presente documento muestra la puesta en marcha de un programa de intervención con evidencia científica en estado de implementación: PACT. Habiéndose avanzado en dos de las fases con 3 niños ya se encuentran evidencias de su beneficio, así como el uso de las estrategias de seguimiento de la motivación del niño, el control del estado emocional, vincularles a estados emocionales de juego y el poner palabras mejoran la atención compartida de los pequeños, su comunicación e intención comunicativa, la aparición de mayor lenguaje, una mayor motivación social y una mayor expresión de afecto.

Palabras clave: TEA; Atención Temprana; motivación social; regulación emocional

\section{ABSTRACT}

Emotion and motivation in tea and early attention. Motivation and emotion are fundamental elements for learning. When we speak of early development and ASD, these two dimensions acquire a greater weight due to socio-emotional deficits, restricted interests and stereotyped behaviors that 


\section{EMOCIÓN Y MOTIVACIÓN EN TEA Y ATENCIÓN TEMPRANA}

prevent children with ASD from being linked in the same way to adults and others. There is wide evidence of the benefits of initiating a specialized intervention in ASD, even though there are only risk signs, that could have an impact on the improvement of the symptoms of the disorder (Dawson, 2008). This document shows the implementation of a program of intervention with scientific evidence in the state of implementation: PACT. Having advanced in two of the phases with 3 children are already evidence of their benefit, as well as the use of strategies to monitor the child's motivation, control of emotional state, link them to emotional states of play and putting words improve the shared attention of the children, their communication and communicative intention, the appearance of greater language, a greater social motivation and a greater expression of affection.

Keywords: ASD; early intervention; social motivation; emotional regulation

\section{INTRODUCCIÓN. ÁREA TEMÁTICA. ANTECEDENTES DE LA TEMÁTICA A TRATAR}

Durante los últimos cincuenta años de investigación y conocimiento acerca del Trastorno del Espectro del Autismo, TEA, el índice de prevalencia se ha incrementado significativamente. Mientras que en 1975 la organización americana Autism Speaks reflejaba un caso de autismo por cada 5.000 nacimientos, en 2008 se hablaba de un caso de TEA por cada 150 y en investigaciones más recientes las cifras son crecientes referenciando que 1 de cada 68 niños podría presentar un TEA (CDC, 2014).

Ante esta creciente situación de prevalencia, la detección y atención temprana se ha convertido en uno de los grandes focos de atención en los últimos 20 años ya que la investigación parece indicar que aquellos menores identificados con TEA tempranamente, que inician un tratamiento precoz, obtendrían mejores resultados en su posterior desarrollo. Así varios grupos profesionales y organizaciones como la Academia Americana de Neurología (Filipek et al., 2000) y la Academia Americana de Pediatría (American Academy of Pediatrics Committee on Children with \& Disabilities, 2001), han defendido la importancia de la detección temprana y habitual en el autismo. Igualmente, en el 2007 las recomendaciones específicas de la Academia Americana de Pediatría (Johnson, Myers, \& American Academy of Pediatrics Council on Children With Disabilities, 2007) incluyeron la vigilancia en cada visita del niño sano, haciendo un especial hincapié en determinadas señales de alarma, así como el seguimiento a hermanos pequeños de niños con TEA.

Existe consenso científico en que cuanto antes empiece la intervención, mejor será el desarrollo del niño, y será posible promover una trayectoria de evolutiva más normalizada, ya que el cerebro antes de los 3 años tiene mucha más plasticidad (Dawson, 2008; Rogers \& Vismara, 2008). De hecho, la literatura científica ha documentado que las experiencias tempranas afectan al desarrollo neurobiológico (Dawson et al. 2012; Knudsen 2004) y algo fundamental y clave en el autismo, que dichas experiencias tienen un efecto de cascada en el desarrollo (Thelen \& Smith 1994). Aún más, se sabe que el primer año de vida, el cerebro se reorganiza para la conexión social, y que este vínculo entre emoción guiado por una motivación social es la base del aprendizaje. En este punto, la evidencia creciente demuestra que el aprendizaje se potencia más cuando está vinculado a actividades que contienen interacciones sociales emocionalmente significativas frente a situaciones donde no existe conexión. (Topál et al. 2008).

En publicaciones recientes se indica que la intervención tiene un gran impacto si sucede antes de los 3 años (CDC, 2009; US.DOE 2011 en Pennington, Cullinan y Southern, 2014) y, en general, si es suficientemente intensiva, puede producir grandes mejoras en $\mathrm{Cl}$ y/o en el comportamiento adaptativo de los niños pequeños con TEA (Eldevik et al. 2009; Reichow, 2012; Virues-Ortega, Rodríguez, \& Yu, 2013). Más aún se ha establecido, que intervenir en los primeros signos de alerta previo a un diagnóstico formal, puede ser más importante e incluso prevenir el comienzo del resto de los síntomas para algunos de los niños (Dawson, 2008). 
A nivel específico las dos grandes áreas de dificultades de las personas con TEA son las dificultades en la reciprocidad socioemocional y los intereses restringidos y estereotipados. Las dificultades socioemocionales pueden mostrarse como una disminución o falta de comprensión de afectos, sentimientos o situaciones compartidas que repercuten en la construcción y comprensión de las situaciones sociales más complejas.

A nivel temprano, se ven afectadas las conductas que regulan las interacciones sociales, apareciendo déficits en conductas de atención compartida y atención conjunta propias del primer año de vida. Así las interacciones sociales pueden variar y estar marcadas por la falta de iniciativa e interés, por hacer iniciaciones sociales peculiares, por falta de respuestas o por no estar coordinadas estas respuestas sociales con otras conductas de comunicación social como la mirada, los gestos y las vocalizaciones. En su estudio de 2011, Mundy establece que el número de conductas de atención conjunta en los menores con TEA es significativamente inferior que los niños con desarrollo típico y en posteriores estudios se establece la relación de la influencia de la atención conjunta en el procesamiento de la información, así como la conexión entre la atención conjunta y los problemas de aprendizaje (Mundy, 2016; Mundy, Sullivan \& Mastergeorge, 2009).

En cuanto a las conductas no verbales que antes se comentaban puede aparecer tanto un contacto ocular escaso 0 , aun existiendo contacto ocular, un fallo en la coordinación y modulación de la mirada con intención comunicativa. También presentan dificultades con los gestos, tanto en su realización como en la comprensión, falta de sincronía de la expresión facial con el contexto, la dificultad para comprender las expresiones faciales y su uso y la utilización del lenguaje corporal. En Ios estudios de indicadores tempranos de TEA con hermanos en riesgo (Zwaigenbaum y col., 2005; 2015) ya encontraron peculiaridades a los 12 meses en contacto ocular, orientación la nombre, imitación, sonrisa e interés social y afectos, todas ellas conductas típicas de los intercambios sociales mediadas y guiadas por la motivación social.

Continuando con las dificultades de reciprocidad socioemocional y conexión también aparecen viejas y tradicionales concepciones erróneas como que los bebes y niños con TEA presenten falta de apego con sus familiares. Existen estudios que muestran, junto con la realidad clínica, que las relaciones de proximidad y el vínculo con los padres existe y es fuerte (Howlin, 1986). Aún más importante en este sentido, es la evidencia del impacto del estilo de interacción entre padres e hijos en los intercambios de los primeros años, que pueden llegar a cambiar en positivo las dificultades de comunicación social que presentaban los pequeños con TEA. Por este motivo, en la actualidad los modelos de atención temprana se están centrando en el trabajo con las familias y el establecer patrones significativos de comunicación social (Hudry et al., 2013), donde la emoción y la motivación sean el elemento central del que parta la interacción y acción conjunta. De hecho, en una reciente investigación se ha postulado que las rutas del apego familiar y el desarrollo social estarían moduladas por una disociación entre las respuestas impulsadas por el apego interno y las dinámicas de aprendizaje atípicas que ocurren durante los episodios de interacción entre el cuidador y el niño que sí son gobernadas por la influencia social y contingencias operantes (Vivanti \& Nuske, 2017). Este hecho nos lleva a mecanismos de intervención en los que esta motivación interna hacia la figura de referencia se generalice y expanda.

Recientemente, en el 2018 la edición de intervención del Diario de Psiquiatría y Psicología Infantil ha revisado la eficacia de los diferentes modelos y programas de intervención específicos en TEA. 48 estudios randomizados, que evalúan 32 modelos, fueron identificados, de los cuales 40 están desde el 2010. De todos los estudios solo 6 cumplían criterios de bajo riesgo, y, en relación con la eficacia de los modelos, el grupo de Green y colaboradores (2015), a través de intervención mediada por los padres con video modelado, si parece enseñar a las padres estrategias de interac- 


\section{EMOCIÓN Y MOTIVACIÓN EN TEA Y ATENCIÓN TEMPRANA}

ción para desarrollar áreas fundamentales del desarrollo como la interacción y la atención conjunta, base de una emoción y motivación compartida. (Green et al., 2015). PACT (Paediatric Autism Communication Therapy) de Aldred y Green es la primera intervención que ha mostrado una reducción significativa en los síntomas de TEA mantenidos durante 6 años (Pickles et al., 2016), siendo la sincronía y la conexión emocional (entendida como la interacción diádica entre el adulto y el niño con una coincidencia en tiempo, intensidad y ritmo) las que generarían otras interacciones sociales que, junto con la atención conjunta, se asociaría a la aparición de lenguaje, elemento central del éxito de este programa.

Es por ello, que la siguiente propuesta de intervención, que se encuentra en fase de implementación, se basa en la puesta en marcha del Programa PACT, centrándose en desarrollar esos momentos de sincronía con emoción y motivación compartida entre adultos de referencia para desencadenar otras áreas de desarrollo como la comunicación, intención comunicativa y la pragmática.

\section{OBJETIVOS DE LA INVESTIGACIÓN}

El objetivo general de este estudio consiste en dar a conocer el Programa de Intervención PACT dirigido a niños con signos de alerta en TEA de edades entre los 2 y los 7 años, demostrando los beneficios de una intervención basada en la emoción y la motivación en la optimización de las competencias comunicativas de los niños con TEA.

Para ello se pretende no sólo analizar los signos tempranos de motivación e interacción social en niños con signos de alerta de TEA y registrar su evolución, sino también los signos de atención conjunta y emoción compartida, a la vez que analizamos los precursores de la comunicación y su desarrollo.

\section{MUESTRA Y/O PARTICIPANTES}

La muestra está formada por 3 niños con edades de 3 años y 2 meses, 3 años y 9 meses y 6 años y 10 meses. Todos los niños están escolarizados en centros ordinarios con apoyos. Los niños de 3 años tienen un diagnóstico de signos de alerta de TEA grave según DSM-V y tras pasar ADOS2 y ADI-R, el niño de 6 años tienen un diagnóstico de TEA sin lenguaje oral. Las familias presentan un nivel socioeconómico medio-alto, formadas por 304 miembros, presentando todas ocupación laboral en, al menos, uno de los progenitores.

\section{PROGRAMA DE INTERVENCIÓN}

PACT es un programa de intervención diseñado para mejorar las competencias de comunicación social en niños con TEA y dificultades del desarrollo del lenguaje. Las bases teóricas de PACT son el resultado de la investigación sobre el desarrollo prelingüístico, pragmático y lingüístico. Este enfoque, se centra en dificultades básicas en materia de atención compartida, comprensión y comunicación intencional que hacen que los niños requieran de un tipo de interacción adaptada, personalizada en función de su nivel individual de comunicación social para su desarrollo. El programa consta de 6 etapas con sus estrategias y propuestas específicas para cada una de ellas. Todas las familias comenzaron en la etapa 1 del programa.

Los principios y metodología en la aplicación del programa serían:

Orientación del desarrollo. La intervención PACT se basa en el desarrollo de habilidades sociales y de comunicación relacionadas con el desarrollo recíproco, social, prelingüístico, pragmático y lingüístico, que son la base de las dificultades en el TEA. El objetivo es guiar a la familia para crear momentos de interacción oportunos y adaptados en el que las respuestas de comunicación y el lenguaje coincidan con la competencia comunicativa del niño. La familia identifica ventanas de oportunidad partiendo de la motivación y los intereses del niño y regulando su nivel emocional, provocando una mayor intencionalidad y un incremento de las interacciones conjuntas. 
Enfoque en las interacciones naturalistas. EI PACT se centra en interacciones diádicas naturalistas que ocurren a lo largo del día a día de una familia para favorecer los momentos de aprendizaje en cualquier situación.

Dirigido por los padres. La intervención establece que familia y profesional son un equipo, estableciendo una relación colaborativa en que, partiendo de las habilidades de la familia, el profesional guía y potencia su iniciativa, su confianza y la toma de decisiones.

Adaptado al estilo de los padres. Teniendo en cuenta y ayudando a reflexionar a las familias sobre su propio estilo de aprendizaje.

Uso del vídeo modelado. De acuerdo con las investigaciones sobre el aprendizaje de adultos y la enseñanza eficaz, el programa de tratamiento emplea el análisis del vídeo y un estilo de terapia reflexivo. Se graban todas las sesiones de juego padre-hijo y, posteriormente, se realiza una sesión de análisis en la que se anima a la familia a identificar episodios satisfactorios de interacción recíproca y a reflexionar sobre su positiva contribución a dichos episodios. Las sesiones analizan la interacción padre-hijo, exploran las señales de iniciación y ayudan a cambiar de enfoque, y pasar de lo que el niño está haciendo a que comprendan que está comunicando, qué le motiva y dónde y cómo está su emoción.

Establecimiento de metas y objetivos específicos. En cada sesión derivados del análisis del vídeo.

Las sesiones son semanales, con una duración entre 1,5 y 2 horas, en las que el padre 0 la madre y el niño realizan la sesión de forma alterna, y en la que se proponen objetivos diferentes para cada miembro de la familia en función del análisis del vídeo realizado, de este modo se interviene en los objetivos de la madre y del padre por separado. Debe existir un compromiso de la familia de establecer una sesión de 30 minutos de juego diario donde se sigue el interés, la motivación y la emoción del niño, ya que éste es el punto de partida, teniendo en cuenta tanto el estado de regulación del niño como la habilidad de la familia para regular en caso de emociones muy intensas.

En cuanto al formato de las sesiones, se realizan en tres fases: (1) Inicial, donde se revisan los avances y se establece la agenda del día; (2) Central, consistente en un periodo de juego entre el padre o la madre y el niño, y grabación durante 10 minutos, para su posterior visionado y análisis, de tal manera que se anotan las conductas observadas así como las observaciones de los padres y la puesta en común sobre las técnicas utilizadas; (3) Final, donde se resume y se registran los objetivos que propone el familiar, así como las actividades e ideas que surgen de forma concreta para la semana. Cabe mencionar que el análisis del vídeo parte de la elección del familiar que decide en qué momento ha disfrutado más y ha sentido que la interacción era más fluida.

\section{RESULTADOS ALCANZADOS}

El programa se encuentra en fase de implementación desde diciembre 2018, encontrándose, las 3 familias, en la etapa 2 del programa. Los resultados del análisis de video-modelado en las conductas de los niños reflejan incrementos en: la motivación del niño en jugar y relacionarse con sus familiares y compartir el juego con otros, conducta que se ha generalizado a la escuela; la emoción positiva del niño medida por el número de veces que inicia un juego conocido, la generalización con otras personas y el número de veces que se ríe a lo largo de las sesiones de video-modelado; la atención a los otros y de la imitación tanto motora como verbal; e incremento y aparición de vocalizaciones y palabras nuevas. A nivel específico los resultados de la etapa 1 del programa aparecen en la tabla 1. 
Tabla 1.

Objetivos individuales medidos en análisis de 10 minutos de video.

\begin{tabular}{|c|c|c|c|}
\hline $\begin{array}{c}\text { ETAPA 1. ESTABLECER } \\
\text { ATENCIÓN COMPARTIDA }\end{array}$ & $\begin{array}{c}\text { NIÑO 1 } \\
3 \mathrm{a} \\
\text { TEA con inicio de } \\
\text { lenguaje oral }\end{array}$ & $\begin{array}{c}\text { Niño } 2 \\
3 \mathrm{a} \\
\text { TEA con palabras }\end{array}$ & $\begin{array}{c}\text { Niño } 3 \\
6^{\text {a }} \\
\text { TEA sin } \\
\text { lenguaje }\end{array}$ \\
\hline $\begin{array}{ll}\text { - } & \text { Atención compartida } \\
\text { - } & \text { Momentos triangulación } \\
\text { mirada }\end{array}$ & $\begin{array}{ll}\text { - } & 8 \text { min } \\
- & 4 \\
- & 5 \text { min } \\
\text { - } & \text { Generalizada } \\
\text { a otros } \\
\text { adultos y/o } \\
\text { niños } \\
\\
\text { Mayor } \\
\text { muestra de } \\
\text { abrazos, } \\
\text { besos y } \\
\text { compartir } \\
\text { juegos } \\
\text { propios. } \\
\text { Incremento } \\
\text { de } \\
\text { vocalizacione } \\
\text { s y repetición } \\
\text { de palabras } \\
\text { nuevas en } \\
\text { contextos de } \\
\text { interacción. }\end{array}$ & $\begin{array}{ll} & 7 \text { min } \\
- & 3 \\
- & \text { Actividades } \\
\text { conocidas } \\
\text { - } & \text { Inicio de } \\
\text { juegos } \\
\text { sociales } \\
\\
\\
\text { Disminució } \\
\text { n momentos } \\
\text { de } \\
\text { desconexió } \\
\text { n o } \\
\text { aparición de } \\
\text { emociones } \\
\text { negativas. } \\
\text { Petición, } \\
\text { comentarios } \\
\text {, preguntas, } \\
\text { negación y } \\
\text { frases más } \\
\text { complejas }\end{array}$ & $\begin{array}{ll}- & 9 \text { min } \\
- & 6 \\
- & \text { Adulto } \\
\text { referenci } & \text { a } \\
- & \text { Mayor } \\
& \text { disfrute } \\
\text { en } \\
\text { interacci } \\
\text { ón adulto } \\
\text { referenci } \\
\text { a. } \\
\text { - Mayor } \\
\text { muestra } \\
\text { de } \\
\text { emoción } \\
\text { comparti } \\
\text { da. } \\
\\
\text { Aparició } \\
\mathrm{n} \\
\text { vocalizac } \\
\text { iones, } \\
\text { sonidos } \\
\text { y } \\
\text { acercami } \\
\text { entos } \\
\text { palabras. }\end{array}$ \\
\hline
\end{tabular}

En cuanto a los objetivos de los familiares se ha encontrado:

Mayor respuesta a las señales contextuales, no verbales y verbales del niño y a interpretar las intenciones del niño.

Seguir la iniciativa, la motivación y la emoción en el juego.

Incremento del tiempo en la espera estructurada

Identificar la respuesta del niño y entender a qué responde esa conducta.

Identificar comportamientos específicos en el juego.

Aprender a reforzar las oportunidades de comunicación.

Adaptar el nivel de apoyo que se da al niño. 
Valorar la intensidad y el momento de la respuesta.

Aprender a dar pistas para fomentar la comunicación y usar tentaciones comunicativas

\section{DISCUSIÓN}

Numerosos estudios se están centrando en determinar modelos de intervención especializados en TEA que produzcan cambios significativos en el posterior desarrollo del trastorno. En ese sentido, en el presente trabajo introduce la implementación de uno de los modelos con mayor evidencia PACT.

Encontrándose el programa en fase de desarrollo, los primeros resultados sí parecen concordar con los resultados de las investigaciones (Pickles et al., 2016) y parecen determinar que estrategias en las que se prima el seguimiento de la motivación del niño, sus intereses y vinculados a emociones positivas en situaciones de atención compartida de juego con el adulto de referencia, potencian un incremento de la atención conjunta, la comunicación, la intención comunicativa y la pragmática.

Estos datos concordarían con los estudios de Charman (2003) y Siller y Sigman (2002) que establecen la importancia de que los padres compartan el foco atencional y emparejen su lenguaje hacia la intención y motivación del niño poniendo palabras a lo que hace. Estas mismas estrategias, junto con la espera, parecen incrementar, igualmente, la intención comunicativa de los niños con TEA hacia sus padres siendo una de las dificultades más documentadas en la literatura científica (Wetherby \& Woods, 2006). Del mismo modo, el programa también potencia la pragmática de los niños produciéndose un mayor uso de la mirada, señalado, gestos, posturas, vocalizaciones y palabras, así como un incremento de las funciones por las que los niños se comunican, más allá de la petición, apareciendo otras funciones como el comentario y la negación, todas ellas dificultades expresadas en niños con TEA (Tavis, Sigman \& Ruskin, 2001).

\section{CONCLUSIONES}

Esta propuesta surge de los trabajos previos que muestran evidencia teórica de la importancia que una intervención temprana, centrada en el desarrollo y donde el niño es el foco de atención, tiene sobre los síntomas de TEA, incluso que los beneficios se mantengan a lo largo de los años. Más específicamente podemos apoyar que la implementación del programa PACT incrementa la motivación del niño hacia los iguales, mantiene un tono emocional positivo en situaciones de interacción, y ello mejora la atención conjunta, la intención comunicativa y el lenguaje.

\section{REFERENCIAS BIBLIOGRÁFICAS}

Aldred, C., Green, J., Howlin, P., Le Couteur, A., Barron, S., Beggs, K., Collino, J., Colmer, R., Houghton, T., Randles, S., Slonims, V., y Taylor, C. (2011). Pre-school Autism Communication Therapy-PACT Manual. Roundway Centre Publishing

American Psychiatric Association. (2013). Diagnostic and statistical manual of mental disorders (5TH Edition). Washington DC: Author.

American Academy of Pediatrics Committee on Children with, \& Disabilities. (2001). The Pediatrician's Role in the Diagnosis and Management of Autistic Spectrum Disorder in Children. Pediatrics, 107(5), 1221-1226. https://doi.org/10.1542/peds.107.5.1221

Charman, T. (2003). Why is joint attention a pivotal skills in autism? Philosophical Transactions of the Royal Society. B:Biological Sciences. 358, 315-324.

Dawson, G. (2008). Early behavioral intervention, brain plasticity, and the prevention of autism spec$\begin{array}{lll}\text { trum disorder. Development and Psychopathology, 203). } & \text {. }\end{array}$ https://doi.org/10.1017/S0954579408000370.

Dawson G, Bernier R, y Ring RH. (2012). Social attention: A possible early indicator of efficacy in 


\section{EMOCIÓN Y MOTIVACIÓN EN TEA Y ATENCIÓN TEMPRANA}

autism clinical trials. Journal of Neurodevelopmental Disorders, 4(1):11. doi: 10.1186/18661955-4-11.

Eldevik, S., Hastings, R. P., Hughes, J. C., Jahr, E., Eikeseth, S., \& Cross, S. (2009). Meta-Analysis of Early Intensive Behavioral Intervention for Children with Autism. Journal of Clinical Child \& Adolescent Psychology, 38(3), 439-450. https://doi.org/10.1080/15374410902851739

Filipek, P. A., Accardo, P. J., Ashwal, S., Baranek, G. T., Cook, E. H., Dawson, G., Kallen, R. J. (2000). Practice parameter: Screening and diagnosis of autism Report of the Quality Standards Subcommittee of the American Academy of Neurology and the Child Neurology Society. Neurology, 55(4), 468-479.

French, L, y Kennedy, E. (2018). Annual Research Review: Early intervention for infants and young children with, or at-risk of, autism spectrum disorder: a systematic review. Journal of Child Psychology and Psychiatry 59:4, pp 444-456.

Green, J., Charman, T., Pickles, A., Wan, M. W., Elsabbagh, M., Slonims, V., Taylor, C., McNally, J., Booth, R., Gliga, T., Jones, E., Harrop, C., Bedford, R., Johnson, M., and BASIS team (2015). Parent-mediated intervention versus no intervention for infants at high risk of autism: a parallel, single-blind, randomised trial. The Lancet. Psychiatry, 2(2), 133-140. doi:10.1016/S22150366(14)00091-1.

Howlin, E. A. (1986). "An overview of social behavior in autisrn" En E. Schopler. \& G.B. Meisvibov (Eds.), Social Behavior in autism. Plenum Press. N.Y.

Hudry, K., Aldred, C., Wigham S, Green J, Leadbitter K, Temple K, Barlow K, y McConachie H; PACT Consortium. (2013) Predictors of parent-child interaction style in dyads with autism. Res Dev Disabil.34(10):3400-10.

Johnson, C. P., Myers, S. M., \& American Academy of Pediatrics Council on Children with Disabilities. (2007). Identification and evaluation of children with autism spectrum disorders. Pediatrics, 120(5), 1183-1215. https://doi.org/10.1542/peds.2007-2361.

Knudsen El. (2004). Sensitive periods in the development of the brain and behavior. Journal of Cognitive Neuroscience, 16:1412-1425. doi: 10.1162/0898929042304796.

Macari, S., DiNicola, L., Kane-Grade, F., Prince, E., Vernetti, A., Powell, K., Fontenelle, S., Chawarska, K. (2018). Emotional Expressivity in Toddlers with Autism Spectrum Disorder. Journal of the American Academy of Child \& Adolescent Psychiatry. Volume 57, Issue 11, November 2018, Pages 828-836.e2.

Mundy P. (2011). The social behavior of autism: a parallel and distributed information processing perspective. In Autism spectrum disorders (eds Amaral DG, Dawson G, Geschwind DH, editors.), pp. 149-171. New York, NY: Oxford University Press.

Mundy, P. (2016). Autism and joint attention: Developmental, neuroscience, and clinical fundamentals. New York City, NY: Guilford Pub. Inc.

Mundy, P., Sullivan, L., \& Mastergeorge, A. M. (2009). A parallel and distributed-processing model of joint attention, social cognition and autism. Autism Research, 2, 2-21.

Pennington, M. L., Cullinan, D., \& Southern, L. B. (2014). Defining autism: variability in state education agency definitions of and evaluations for autism spectrum disorders. Autism research and treatment, 2014, 327271. doi:10.1155/2014/327271.

Pickles, A., Le Couteur, A., Leadbitter, K., Salomone, E., Cole-Fletcher, R., Tobin, H., Gammer, I., Lowry, J., Vamvakas,G., Byford,S., Slonims, V., McConachie, H., Howlin, P., Parr,J.R., Charman,T., Green,J., \& Aldred, C. (2016). Parent-mediated social communication therapy for young children with autism (PACT): long-term follow-up of a randomised controlled trial. The Lancet, 388(10059), 2501-2509. 
Reichow, B. (2012). Overview of Meta-Analyses on Early Intensive Behavioral Intervention for Young Children with Autism Spectrum Disorders. Journal of Autism and Developmental Disorders, 42(4), 512-520. https://doi.org/10.1007/s10803-011-1218-9.

Rogers, S. J., \& Vismara, L. A. (2008). Evidence-based comprehensive treatments for early autism. Journal of Clinical Child and Adolescent Psychology: The Official Journal for the Society of Clinical Child and Adolescent Psychology, American Psychological Association, Division 53, 37(1), 8-38. https://doi.org/10.1080/15374410701817808

Schreibman, L., Dawson, G., Stahmer, A. C., Landa, R., Rogers, S. J., McGee, G. G., Kasari, C., Ingersoll, B., Kaiser, A.P., Bruinsma, Y., McNerney, E., Wetherby, A., y Halladay, A. (2015). Naturalistic Developmental Behavioral Interventions: Empirically Validated Treatments for Autism Spectrum Disorder. Journal of Autism and Developmental Disorders, 45(8), 2411-2428. doi:10.1007/s10803-015-2407-8.

Siller, M y Sigman, M. (2002). The behaviors of parent of children with autism predict the subsequent development of their children communication. Journal of Autism and Developmental Disorders, 32, 77-89.

Tavis, T., Sigman, M., y Ruskin, E. (2001). Links between social understanding and social behaviour in verbally able children with autism. Journal of Developmental Disorders, 31, $2119-130$.

Thelen E, Smith L. (1994) A dynamic systems approach to the development of cognition and action. Boston: MIT Press.

Topál J, Gergely G, Miklósi Á, Erd hegyi Á, Csibra G. (2008). Infants' perseverative search errors are induced by pragmatic misinterpretation. Science, 321(5897):1831-1834. doi: 10.1126/science.1161437.

Virues-Ortega, J., Rodríguez, V., \& Yu, C. T. (2013). Prediction of treatment outcomes and longitudinal analysis in children with autism undergoing intensive behavioral intervention. International Journal of Clinical and Health Psychology, 13(2), 91-100. https://doi.org/10.1016/S16972600(13)70012-7.

Vivanti, G., y Nuske, H.J. (2017). Autism, attachment, and social learning: Three challenges and a way forward. Behavioural Brain Research, 325 (B), 251-259.

Wetherby, A. M., y Woods, J. J. (2006). Early social interaction project for children with autism spectrum disorders beginning in the second year of life: A preliminary study. Topics in Early Childhood Special Education, 26, 67-82.

Zwaigenbaum, L., Bryson, S., Rogers, T., Roberts, W., Brian, J., Stamari, P. (2005). Behavioral manifestations of autism in the first year os life. International Journal of Developmental Neuroscience 23, 143-152.

Zwaigenbaum, L., Bauman, M. L., Choueiri, R., Kasari, C., Carter, A., Granpeesheh, D., Mailloux, Z., Smith Roley,S., Wagner, S., Fein, D., Pierce, K., Buie, T., Davis, P., Newschaffer, C., Robins, D., Wetherby, A., Stone, W. L., Yirmiya,N., Estes, A., Hansen, R. L., McPartland, J. C.., y Natowicz, M. R. (2015). Early Intervention for Children with Autism Spectrum Disorder Under 3 Years of Age: Recommendations for Practice and Research. Pediatrics, 136(Supplement 1), S60-S81. https://doi.org/10.1542/peds.2014-3667E. 
